\title{
Regresar a casa: análisis de la primera temporada de Homeland (Michael Cuesta, 2011)
}

\begin{abstract}
Aarón Rodríguez Serrano*
Recibido: mayo 20 de 2014 - Aceptado: septiembre 13 de 2014

\section{Resumen}

El presente trabajo centra su atención en la primera temporada de la serie de televisión Homeland (Michael Cuesta, 2011) para realizar un análisis sociológico y político de su contenido. Para ello, se utilizará una metodología de análisis textual proveniente de las ideas políticas del pensador Slavoj Zizek, concretamente, de sus escritos en torno al trauma norteamericano pos $11 \mathrm{~S}$. Del mismo modo, se trabajarán las conexiones que se establecen entre ley, cuerpo e ideología en el tapiz narrativo televisivo.
\end{abstract}

Palabras clave: Homeland, primera temporada, política, análisis textual.

* Doctor en Comunicación Audiovisual. Profesor Titular en la Universidad Europea de Valencia, en el ámbito del Análisis de la imagen. Correo electrónico: aaron_stauff@hotmail.com 


\title{
Back home: analysis of the first season of Homeland (Michael Cuesta, 2011)
}

\begin{abstract}
This article is focused on the first season of TV series Homeland (Michael Cuesta, 2011), intended to make a sociological and political analysis of its content. For this purpose, a textual analysis methodology will be used taken from the political ideas of thinker Slavoj Zizek, specifically from his written work about the North American trauma after the 11S. In the same way, connections among law, body, and ideology in the tapiz narrative televisivo are addressed.
\end{abstract}

Key words: Homeland; first season; politics; textual analysis. 


\section{Introducción. Metodología y objeto de estudio}

En el momento de redactar estas líneas, el análisis textual audiovisual está especialmente interesado en la importancia, cada vez mayor, que las series de televisión están tomando en la construcción de los sujetos. Lo que se ha denominado una "segunda edad dorada" de la televisión (Thompson, 1996; Cascajosa, 2005) ofrece estimulantes y complejos retos que tienen que ver tanto con las modificaciones en la recepción del texto', como con la posible lectura ideológica que se realice de los mismos. Las series parecen encajar con los hábitos del sujeto "líquido" de Bauman (2006), al no exigir ningún espacio ni ningún tiempo prefigurado para su disfrute: sus capítulos se insertan con comodidad en las hendiduras que dejan las obligaciones productivas de la empresa, que pueden ser utilizadas como nexo social -el fenómeno Lost (J. J. Abrahams, 2004-2010) es un magnífico ejemplo de cómo generan auténticas comunidades hermenéuticas-, y por supuesto, ofrecen un cierto retorno al relato "clásico" que una parte importante del cine contemporáneo parecía haber perdido (González Requena, 2006). Allí donde la modernidad fílmica derrumbó los usos y costumbres de un cierto Hollywood (Font, 2002), la serie contemporánea funciona como el retorno de lo reprimido, la resurrección de una manera de construir relatos actualizando y respetando las normas de la narrativa clásica.

Productos puramente distópicos como Twin Peaks (David Lynch, 1990-1991) son más bien excepciones ante un panorama general que sigue bebiendo de los géneros clásicos -con las típicas modificaciones postmodernas, por supuesto-y apoyándose sobre estructuras que garantizan la recepción espectatorial en unas coordenadas no muy diferentes de las enarboladas por el Modo de Representación Institucional (Gómez Tarín, 2011). Rasgos como la transparencia enunciativa o la construcción de personajes más o menos reconocibles (Bordwell, Staiger y Thompson, 1997) forman parte de la inmensa mayoría de los productos ofrecidos por las cadenas de televisión estadounidenses, lo que como ya sabemos, implica también una serie de rasgos ideológicos.

Ahora bien, ¿cómo influye esta nueva hornada de productos audiovisuales en la metodología analítica tradicional? En primer lugar, por su propia naturaleza, las series de televisión suelen ser presentadas como textos abiertos, cuya duración en pantalla depende tanto de los resultados de audiencia como de la capacidad de los guionistas para mantener el interés sin defraudar las expectativas de los espectadores. En segundo lugar, la naturaleza extensa de las producciones dificulta notablemente la ejecución de técnicas microanalíticas (Zunzunegui, 1996), recomendando en su lugar un análisis transversal que acote el objeto de estudio ya sea por temporadas o incluso por capítulos. La evolución de los arcos de transformaciones de los personajes -cuyos constantes cambios, contradicciones y descubrimientos garantizan una gran parte del sabor narrativo de una serie- hace que las convenciones sobre el guion tradicional (McKee, 2002 y Sánchez-Escalonilla, 2001)

1 La serie de televisión, por su propia naturaleza, es un espectáculo necesariamente escindido cuya recepción "normativa" se despliega en el tiempo, torsionando las tramas y dependiente de una estructura particular mediada por la inserción de publicidad o por la necesidad de fidelizar a la audiencia (Calabrese, 1994). No deja de ser, por tanto, un mecanismo narrativo propio del discurso televisivo (González Requena, 1999). 
sean constantemente puestas en duda, alejándose de la tradición estructural aristotélica para generar una suerte de movimiento líquido incontrolable e impredecible.

Por estos motivos, hemos decidido acotar nuestro estudio al análisis de la primera temporada de la serie Homeland (Michael Cuesta, 2011), privilegiando una mirada transversal en la que nos valdremos de las herramientas ideológicas que el filósofo Slavoj Zizek ha propuesto en las últimas décadas para despejar las aristas ideológicas y comprobar cómo la serie se inserta en un panorama de crisis pos 11S, contribuyendo al debate sobre las relaciones entre sujeto, poder y eficacia simbólica. Del mismo modo, pensamos que el cierre de la tercera temporada (recién emitido en el momento de redactar el presente trabajo) clausura también una cierta unidad narrativa que privilegia la posibilidad de mirar hacia el pasado para comprender cómo se sedimentaron ciertos estilemas que, peor que mejor, fueron simplemente completados durante las dos temporadas posteriores.

\section{Homeland: escrituras del trauma postmoderno}

La primera temporada de Homeland se propone como una rescritura de la serie israelí Prisioners of war (Hatufim, Gideon Raff, 2009-2012). La localización del texto base es fundamental para entender el origen de dos productos que se enfrentan, desde distintas perspectivas, ante un trauma ideológico que localiza al Otro Islámico en su centro. En el caso norteamericano, responde a una necesidad de forma narrativa ante la reciente generación de un trauma exterior (la reconfiguración de las relaciones internacionales tras las acciones bélicas en Afganistán e Irak) y de un trauma interior (el pánico desatado tras los atentados del 11S, pero también el descubrimiento de la fragilidad democrática en el monstruo bicéfalo Guantánamo/Abu Ghraib). En un momento de profunda crisis ideológica, la construcción de relatos acude para generar textos que suturen y den forma a los traumas (C. Lindy, 2002; Vila, 2004), y en ellos se establece un debate, una lucha de tensiones que señala los puntos ciegos del sistema ideológico.

En efecto, Homeland parte de una dialéctica entre las dos opciones mayoritarias que se despliegan en el panorama político internacional, esto es, de dos leyes cuyo enfrentamiento bélico arrastra necesariamente la vida de los sujetos. De un lado el sistema norteamericano -escindido, a su vez, en tres grandes esferas conectadas entre sí: el brazo militar, los organismos políticos, y las redes familiares que trenzan las relaciones sociales en Occidente-, y del otro, esa masa confusa de cargos y acciones que representa el fundamentalismo islámico. Frente a la claridad con la que se plantean nuestras jerarquías y las tensiones de poder, el terrorismo exterior es retratado como Otro ${ }^{2}$ polimorfo e indefinible. Ambas leyes, a su vez, están literalmente atravesadas y justificadas por el trauma: el inminente

2 Cuando utilizamos la expresión "Otro", no nos referimos únicamente a la alteridad, a ese segundo que parece exigirnos una posición existencial que nos invita al diálogo (Habermas, 1981), o en el límite, a un hipotético encuentro con Dios mediado por una ética (Begrich, 2007). Antes bien, para referirnos al Otro islámico nos basamos en la reinterpretación lacaniana del término propuesta por Zizek, basada en el conflicto y en el choque simbólico de goces y presencias en la esfera local. El "Otro" en Homeland sólo puede ser leído en esta dirección, ya que su problemática es, muy precisamente, su infiltración en nuestras estructuras. 
ataque terrorista que planea sobre las sociedades del primer mundo, los "ataques preventivos" contra la población civil en las zonas de conflicto orientales. La reconciliación, el diálogo, el encuentro entre ambos sistemas legislativos es una posibilidad narrativa que ni siquiera se contempla en la primera temporada de la serie. El Otro es asumido así en su total radicalidad, escapando de posibles tentaciones "políticamente correctas" para alcanzar el corazón del conflicto. Desde el momento en el que Homeland construye un Otro indomable e incomprensible, está evitando también la tentación de "normalizarlo", de "colonizarlo", de convertirlo en una pulpa fácilmente digerible por el espectador. Su lugar simbólico es necesariamente inefable, exterior - ellos, que no son como nosotros-, lo que le otorga el poder de la diferencia, y por eso mismo, la verosimilitud narrativa y la dignidad de la supervivencia. Volveremos sobre esta idea cuando encaremos el análisis de la posición simbólica de Issa, el hijo del líder terrorista Abu Nazir.

En cualquier caso, bajo la Ley, se encuentran los cuerpos. Cuerpos vinculados a las estructuras de poder (Foucault, 1986), en los que se escribe literalmente el trauma ideológico. ¿Cuál es el punto de partida de la serie? El descubrimiento de un cuerpo, el del Sargento Brody (Damian Lewis), que tras ocho años de secuestro por una célula de Al-Qaeda es rescatado por el ejército estadounidense. Los interrogantes activos que se le plantean al espectador están, necesariamente, conectados con las relaciones entre cuerpo y poder: ¿̇sigue perteneciendo ese cuerpo a nuestra red simbólica? ¿Ha podido ser manipulado, convertido en una herramienta para el enemigo, "reprogramado" para atentar contra nuestra propia sociedad? Brody es un cuerpo-máquina (Deleuze y Guattari, 1998), un cuerpo que actúa como repositorio para un cierto saber político y, por lo tanto, como una posible arma arrojadiza contra el enemigo. El cuerpo de Brody se exhibe en la televisión como el triunfo de Occidente, como el hijo pródigo que retorna tras una estancia en el infierno del Otro con sus convicciones intactas.

Los guionistas organizan inteligentemente la gestión de la información en los primeros capítulos convirtiendo la vida de Brody en un espectáculo virtual multipantalla. De un lado, las cámaras de la prensa que convierten al personaje en un ser uniformado -cuerpo del ejército- y retransmiten sus discursos - su palabra impostada - para una audiencia que carga con su trauma, y del otro, mediante la vigilancia intensiva que la agente Carrie (Claire Danes) impone ilegalmente sobre su hogar llenándolo de microcámaras y micrófonos. Brody apenas es otra cosa que un sujeto-para-la-contemplación. Es escrutado por la opinión pública (frente a la que finge), y también por la Ley en la intimidad de su retorno a la familia (frente a la que, como sabremos, también finge). Su vida es una impostación total: la división entre esfera pública y privada, como corresponde a la modernidad líquida, ha desaparecido (Bauman, 1999), y en su lugar lo único que queda es una superposición de mascaradas, de rituales, de rostros intercambiables que se aferran brutalmente a palabras deshilvanadas -Ejército, Patria, Familia, Comunidad... curiosamente, las categorías "zombies" de Ulrich Beck (2002)- para no derrumbarse. Brody se ofrece como el relato vivo, el storytelling total que garantiza la supervivencia ideológica y la buena salud del Poder (Salmon, 2008). 
El cuerpo se ofrece a esa contemplación -vaciada de todo sentido- mediante un ritual de aceptación social que el dispositivo televisivo traduce en un juego de puestasen-abismo puramente posclásicas (Palao Errando, 2012, 100). Cada espacio en el que se posiciona se convierte en un lugar de espectáculo, un lugar que parece exigir un comportamiento determinado y una localización simbólica, un no-lugar (Augé, 2004) desprovisto de cualquier verdad. Frente a esta topografía alienante, Brody dispone de un punto ciego, de un rincón propio en el que puede realmente ser: su garaje. Michael Cuesta utiliza una estrategia ya aplicada en su anterior serie Dexter ${ }^{3}$ para mostrar el magma de dolor y de angustia que rodea al protagonista. En esta ocasión, el "punto ciego" de las cámaras es, por extensión, el lugar de lo sagrado, el lugar privilegiado del Otro en el que el soldado estadounidense se refugia para realizar la oración islámica.

Y bien, merece la pena detenerse sobre esta idea. Brody exhibe frente al espectador de la serie toda una serie de conductas distópicas relacionadas con el cuerpo que el espectador puede "asimilar" como consecuencia directa de su trauma como prisionero. Así, no solo su piel está completamente arrasada por las cicatrices de las torturas y las palizas -una topografía del horror político del Otro-, sino que, además, su conducta sexual se presenta como claramente no-normativa. En su primer encuentro con su esposa acomete poco menos que una violación, mientras que en el segundo se limita a masturbarse sobre ella, reduciéndola a una concepción puramente fantasmática, un objeto total. Sin embargo, el verdadero descubrimiento que cambia el rumbo de la serie no está en esta esfera de lo íntimo/sexual, sino en la problemática de la creencia, de la oración: En el momento en el que observamos cómo un sargento de los marines estadounidenses presta su cuerpo a la oración islámica, observamos el cortocircuito ideológico total entre los dos bloques de poder.

\section{La palabra de Casandra: Carrie Mathison}

En el polo opuesto al Sargento Brody, los guionistas de la serie colocan a la protagonista femenina, Carrie Mathison (Claire Denis). Al contrario que el recién recuperado marine, Carrie no tiene una red familiar desde la que construirse. Y, sin embargo, también está escindida. Frente al filo militar/familiar - esto es público/íntimo- de Brody, Carrie propone una división entre lo profesional (meticulosa y entregada agente de la CIA) y personal (enferma mental que se arroja a relaciones efímeras con desconocidos). La conexión entre el trauma político y el goce sexual aflora de nuevo, si bien con un interesante matiz que tiene mucho que ver con la evolución de los roles sexuales. Brody es el héroe militar, marido y esposo que cimienta la tradición norteamericana, y se manifiesta como quebrado en lo sexual y terrorista islámico en lo íntimo. Claire, por su parte, recoge el perfil contemporáneo de mujer emancipada, sin esposo ni hijos, trabajadora infatigable y sexualmente satisfecha y autónoma -enferma mental y desesperada en la otra cara de su personalidad-.

3 Dexter, al igual que el Sargento Brody, es un personaje obligado a realizar un fingimiento constante en torno a la Ley política (su "ser policía") y a la Ley familiar (su "ser padre"). En esa ocasión, se vale de un pequeño cobertizo junto a la casa en el que apila los resultados de sus investigaciones y los trofeos de sus asesinatos. 
Un dato interesante de Homeland es que no esconde una visión conservadora de lo femenino: ambos cánones tradicionales de género están arrasados por igual, completamente erosionados desde la raíz. La lectura de la serie parece más bien apuntar a una caducidad crónica de los arquetipos, a la imposibilidad que las antiguas mallas simbólicas normativas heterosexuales demuestran a la hora de arropar las conductas de los sujetos. Antes bien, lo que se rebela en el interior de los cuerpos es ese malestar que estamos cartografiando en el artículo entre el cuerpo y la ideología: la aceptación voluntaria del exceso, ya sea por la vía del terrorismo o de la autodestrucción.

El síntoma, en Homeland, se dispara precisamente ante un saber relacionado con la crisis de la Ley. Brody se convierte en un fanático cuando comprende que las acciones militares estadounidenses responden a una lógica monstruosa y aberrada. Carrie es poseída por su brote cuando la CIA decide expulsarla, precisamente por encontrar la verdad y comprender la amenaza. Ambos están hermanados por el saber excesivo, por la traición sufrida a manos de las instancias simbólicas que habían dirigido su actividad profesional. De hecho, Carrie se hermana con una larga tradición de figuras femeninas atravesadas entre la profecía, el saber y la locura que se remonta a Casandra, pasa de puntillas por Juana de Arco y desemboca en la Justine de Melancholia (Lars von Trier, 2011). La Palabra de la mujer, por muy verdadera que sea, es desoída voluntariamente por los altos cargos del poder, arrojándola a la esfera del delirio, de la alucinación, de la enfermedad.

\section{La palabra del Padre}

La figura de Brody encara uno de los grandes problemas del Occidente contemporáneo: ¿qué significa ser un padre? Su personaje se entronca perfectamente en esa visión explicitada por Gil Calvo:

Es lo que estamos viendo actualmente en la contemporánea sociedad posindustrial donde a escala privada predomina la figura del "padre ausente" y a escala pública también se asiste al retiro del patriarca, manifestado por el descrédito de la política, el deterioro de las instituciones y el declive de la sociedad civil (GIL CALVO, 2006, 260-261).

Doble rostro, doble crisis cuya salida parece encontrarse directamente conectada al terror social. Si la profecía delirante de Carrie -la única que realmente sabe que Brody es un terrorista que se dispone a atentar contra toda la cúpula política norteamericana- no se cumple, no es por su falta de exactitud, sino porque los guionistas utilizan la introducción de una tradicional salvación en el último minuto, gracias a la hija del Sargento.

Sin embargo, y frente a lo que se pudiera creer, no se trata de un Deus ex machina tradicional, ni de un parche aplicado para justificar la continuidad de la serie. Antes bien, nos atrevemos a proponer que todo Homeland puede ser leído como una exploración de las relaciones paternofiliales a través de la palabra. Es necesario recordar el tercer seminario de Jacques Lacan, con su introducción de la noción del Nombre-Del-Padre y su voluntad de que el padre sea, ante todo, palabra. 
La figura de Brody bascula entre dos posiciones paternales simbólicas atravesadas por la catástrofe. En primer lugar, como ya hemos comentado, es situado en esa posición por el propio Abu Nazir cuando le encarga la educación de su hijo Issa en el capítulo 9. Su función paterna "sustitutiva" es designada por el máximo representante del Otro, el líder ideológico definitivo. Todo el capítulo está construido en torno a una estructura de aproximación entre Brody y el pequeño, que en ningún caso debería ser considerada como una metáfora del entendimiento entre dos culturas. Antes bien, lo que el Marine propone es ni más ni menos que un proceso de enculturación soterrado donde, además de enseñar a Issa su idioma, intenta impregnarle del modo de vida occidental. La prueba evidente es que los nexos que conectan ambas figuras están directamente vinculados con el espectáculo y el deporte. En un proceso similar a la propia construcción que Hollywood realiza sobre el resto del mundo, Brody utiliza un curioso operador textual como conector (una pelota de fútbol) para después obligarle a recitar un himno deportivo.

La palabra de Brody, por mucho que esté falsamente dirigida por una conexión emocional, debe ser leída como una suerte de injerto cultural, el intento de arrancar a Issa de las garras de ese Otro misterioso y totalitario para convencerle de los "sanos valores" de la democracia norteamericana, y por supuesto, de sus mecanismos espectaculares. Cuando el pequeño muera en un ataque estadounidense, se demostrará el poco sentido, la fragilidad del mensaje transmitido. La verdad, el núcleo obsceno de la Ley occidental, se concreta en la acción desoladora de las bombas, el desgarro, el cadáver. Paternidad frustrada, por lo tanto, en el altar del mismo sistema simbólico que se pretendía defender.

Posteriormente, el Sargento regresa a su hogar para encontrarse con dos hijos -en esta ocasión, biológicos, hijos en lo Real- que deben asumir su presencia después de ocho años. El viejo problema narrativo del retorno del héroe (Propp, 2000; Campbell, 1959) es reinterpretado aquí en clave de traición y de extrañamiento. Los hijos ya contaban con un Padre Simbólico que ocupaba su lugar -el marine Mike Faber (Diego Klattenhoff)-, que de pronto será destronado por ese cuerpo desconocido que ocupa su lugar no solo en su constelación educativa, sino en el lugar del deseo de su madre. Nos encontramos en un terreno puramente melodramático que los guionistas explotan generando una frágil complicidad entre Dana (Morgan Saylor), la hija mayor, y su recién recuperado padre.

Dana está construida a medio camino entre el núcleo familiar y una confusa épica de rebelión adolescente -consume drogas blandas-y parece no encajar en los mecanismos de puesta en escena espectacular que quieren convertir a Brody en un héroe nacional. Su situación en el tablero dramático tiene más que ver con una ciudadanía escéptica que se niega a plegarse a las líneas dominantes de la ideología: tolera -aunque con un miedo reverencial- la relación de su padre con el Islam, intuye los problemas y las mentiras que laten en todos los personajes, no se conmueve ante la historia de la Guerra Civil estadounidense. En el límite, representa a todo un sector de espectadores que enarbolan una duda razonable frente al discurso del patriotismo y de la Ley.

De ahí que sea precisamente ella la clave necesaria para enfrentarse con la amenaza total del Otro. Si anteriormente señalábamos que no existía en Homeland un discurso 
de la reconciliación Occidente/Oriente, ahora debemos matizar que la posibilidad de la cooperación solo puede tener lugar en el seno de la propia comunidad norteamericana. En este sentido, nuestra serie funciona en paralelo a otras propuestas contemporáneas como Margaret (Kenneth Lonergan, 2011) o incluso United 93 (Paul Greengrass, 2006) que intentan generar fábulas sobre la necesaria reconciliación de la sociedad estadounidense en la lucha contra el fundamentalismo. Dana aporta la medida exacta de crítica como para situarse fuera de la esfera del poder, pero también la necesaria dosis de compromiso -en este caso, familiar-como para permitir que el bien se imponga. Y aquí, debemos recordarlo, es precisamente el nexo familiar el único que sigue funcionando cuando todos los demás -el religioso, el político, el militar- han desaparecido. Y lo hace bajo la fórmula de una promesa. Como señalábamos, cuando el Sargento Brody está a punto de inmolarse, es obligado por su hija a realizar una única promesa: volverá a casa. Retornará al hogar. Recuperará su posición como Padre Simbólico. Y la importancia total de este momento -sin duda, el clímax narrativo al que conducen los once episodios anteriores- solo puede ser leída en la inmensa resonancia metafórica que encierra. La promesa del retorno al hogar tiene un contenido inscrito, incluso, en el título de la serie (Home-land, lo que se puede traducir como la tierra a la que uno pertenece, su hogar de origen), lo que entraña una profunda transformación de los miedos y las posiciones políticas. Brody debe volver, dejando atrás esa deuda simbólica que encierra el cadáver de Issa, cuerpo muerto que representa el

[...] "fantasma fundamental de la cultura de masas contemporánea", ese fantasma del retorno del muerto vivo: el fantasma de una persona que no quiere estar muerta y retorna amenazante una y otra vez (...) El retorno de los muertos es signo de la perturbación del mito simbólico, del proceso de simbolización; los muertos retornan para cobrar alguna deuda simbólica impaga (Zizek, 2000, pp. 47-48).

Issa es utilizado por Abu Nazir para rescribir la deuda, una deuda que solo se puede saldar con la destrucción de la cúpula política. Dana, a su vez, se posiciona en el lugar de la deuda, atorándola, exigiendo en su lugar un acto que le permita a su padre regresar del territorio del trauma. Esto es, que realmente asuma las implicaciones del Nombre-DelPadre. O si se prefiere, "la promesa, en el fondo, de que hay un relato para el hijo, de que es posible, para él, una experiencia de lo real que tenga sentido" (Casanova, 2010, p. 100).

\section{Conclusiones}

A lo largo de las presentes páginas hemos intentado realizar una primera aproximación a los problemas principales que se plantean en torno a la primera temporada de la serie televisiva Homeland. En primer lugar, hemos encarado algunos de los rudimentos concretos contextuales - procesos de recepción, metodología de análisis-, que sin duda nos permitirán ampliar esta línea de investigación en futuras publicaciones.

En segundo lugar, hemos realizado algunos apuntes sobre el análisis propiamente textual, siguiendo la división Ley/cuerpo/palabra, y generando un encuentro dialéctico entre los dos protagonistas principales de la serie. Esto nos ha permitido observar no solo las aristas ideológicas de los dos sistemas de ley propuestos -las democracias liberales 
frente al fanatismo islámico-, sino las consecuencias que dicha dialéctica provoca en los sujetos que están vinculados con ella. La división entre cuerpo y palabra nos ha llevado directamente al centro de nuestra investigación: el malestar expuesto en la serie que los sujetos parecen experimentar al encontrarse tanto con su goce como con la relación simbólica que proyectan hacia los otros.

Obviamente, el límite principal de nuestra investigación es la propia naturaleza temporal del texto acotado. A lo largo de los próximos años, y si el público sigue apoyando su difusión, las tramas narrativas de Homeland seguirán complicándose y podrán encarar nuevos y estimulantes problemas. En la imagen final que cierra la primera temporada contemplamos cómo Carrie, perdida en mitad de su delirio, intenta recordar el nombre de Issa, esto es, la deuda simbólica impaga, el lugar en el que la Palabra de Brody se atoró y se traicionó a sí misma. Sin duda, es una imagen poderosa que resume con precisión muchos de los focos del malestar posmoderno en Occidente: la quiebra de lo simbólico, el desgarro de la estabilidad mental, la presencia de Otro amenazante, la necesidad de encontrar una palabra que nos permita, de alguna manera, regresar a casa.

\section{Bibliografía}

Augé, M. (2004): Los "no lugares", espacios para el anonimato: una antropología de la sobremodernidad. Barcelona: Gedisa.

Bauman, Z. (1999): Modernidad líquida. Buenos Aires: Fondo de Cultura Económica.

Bauman, Z. (2006): Vida líquida. Barcelona: Paidós.

Beck, U. (2002): Libertad o capitalismo. Conversaciones con Johannes Willms. Barcelona: Paidós.

Begrich, A. (2007): "El encuentro con el otro según la ética de Levinas". Teología y cultura, vol. 7, pps. $71-81$.

Bordwell, D., Staiger, J. y Thompson, K. (1997): El cine clásico de Hollywood: estilo cinematográfico y modo de producción hasta 1960. Barcelona: Paidós.

C. Lindy, David (2002): "Holocaust Movies: Watching the unwatchable". Journal of Applied Psychoanalytic Studies, Vol. 4, pp. 127-133.

Campbell, J. (1959): El héroe de las mil caras: psicoanálisis del mito. México: Fondo de Cultura Económica.

Casanova, B. (2010): "Relato y fin del mundo. The day after tomorrow". Revista Trama E Fondo, N. ${ }^{\circ} 29$, pp. 95- 102 .

Calabrese, O. (1994): La era neobarroca. Madrid: Cátedra.

Cascajosa, C. (2005): "Por un drama de calidad en la televisión: la segunda edad dorada de la televisión norteamericana". Comunicar: Revista Científica Iberoamericana de comunicación y educación. Número 25. (CD-ROM).

Deleuze, G. y Guattari, F. (1998): El anti Edipo. Capitalismo y esquizofrenia I. Barcelona: Paidós.

Font, D. (2002): Paisajes de la Modernidad: Cine Europeo, 1960 1980. Barcelona: Paidós.

Foucault, M. (1986): Vigilar y castigar. Nacimiento de la prisión. Madrid: Siglo XXI.

Gil Calvo, E. (2006): Máscaras masculinas. Héroes, patriarcas y monstruos. Barcelona: Editorial Anagrama.

Gómez Tarín, F. (201 1): Elementos de narrativa audiovisual: expresión y narración. Cantabria: Editorial ShangriLa. González Requena, J. (1999) El discurso televisivo: espectáculo de la posmodernidad. Madrid: Cátedra. 
González Requena, J. (2006) Clásico, manierista, postclásico: los modos del relato en el cine de Hollywood. Valladolid: Castilla Ediciones.

Habermas, J. (1981) Teoría de la acción comunicativa I y II. Madrid: Taurus.

Mckee, R. (2002) El guión: sustancia, estructura, estilo y principios de la escritura de guiones. Barcelona: Alba.

Palao Errando, J. A. (2012) "Hiperencuadre/Hiperrelato: Apuntes para una narratología del film postclásico". Revista Comunicación, N. ${ }^{\circ} 12$, pp. 94-114.

Propp, V. (2000) Morfología del cuento. Madrid: Fundamentos.

Salmon, C. (2008): Storytelling. La máquina de fabricar historias y formatear las mentes. Madrid: Península.

Sánchez-Escalonilla, A. (2001) Estrategias del guion cinematográfico. Barcelona: Ariel.

Thompson, R. J. (1996): Television 's second Golden age: From "Hill Street Blues" to "ER". Nueva York: Continuum. Vila, S. (2004): Deseo en la imagen: cine y fantasma en el 11 de Septiembre. Valencia: Editorial Aletheia.

Zizek, S. (2000): Mirando al sesgo. Una introducción a Jacques Lacan a través de la cultura popular. Buenos Aires: Paidós.

Zunzunegui, S. (1996): La mirada cercana. Microanálisis fílmico. Barcelona: Paidós. 
